\title{
DESENVOLVIMENTO COGNITIVO, LINGUÍSTICO E HISTÓRICO DE RISCO PSÍQUICO EM CRIANÇAS DE 2 ANOS
}

\section{Maria Fernanda Almeida Silva}

Mestre em Psicologia pelo Programa de Pós-Graduação em Psicologia na Universidade Federal de Santa Maria (UFSM), Brasil.

\section{Inaê Costa Rechia}

Pós-Doutorado em Distúrbios da Comunicação Humana pela Universidade Federal de Santa Maria (UFSM), Departamento de fonoaudiologia, Brasil.

\section{Sabrina Felin Nunes}

Doutoranda em Distúrbios da Comunicação Humana pelo Programa de Pós-Graduação em Distúrbios da Comunicação Humana na Universidade Federal de Santa Maria (UFSM), Brasil.

\section{Ana Paula Ramos de Souza}

Pós-Doutorado em Letras pela Universidade Federal do Rio Grande do Sul (UFRGS). Docente do Programa de Pós-Graduação em Psicologia pela Universidade Federal de Santa Maria (UFSM), Brasil.
RESUMO: Analisar a concordância entre o roteiro "Indicadores de Referência ao Desenvolvimento Infantil" (IRDI) e o Modified Checklist for Autism in Toddlers (M-CHAT) na detecção de risco psíquico, bem como investigar a possível relação entre risco psíquico e alterações no desenvolvimento cognitivo e na aquisição da linguagem. Avaliaram-se 20 bebês entre um e 24 meses, em pesquisa de coorte, a partir dos instrumentos IRDI (dos 04 meses incompletos aos 18 meses), M-CHAT e Bayley III (18 e 24 meses). Houve correlação estatisticamente significante $(p=0,004)$ entre alteração de linguagem no Bayley III e M-CHAT aos 18 meses. Observaram-se, ainda, crianças com risco psíquico (IRDI-18 meses) e baixo desempenho no teste Bayley III. Observou-se ausência de concordância entre IRDI e M-CHAT. Por outro lado, a análise entre risco no M-CHAT e alterações de linguagem na escala Bayley III evidenciaram as graves limitações linguísticas em casos de risco para autismo.

PALAVRAS-CHAVE: Desenvolvimento infantil; Cognição; Linguagem; Risco; Transtorno autístico.

\section{COGNITIVE AND LINGUISTIC DEVELOPMENT AND PSYCHIC RISK HISTORY IN T WO-YEAR-OLD CHILDREN}

\begin{abstract}
Current study analyzes agreement between the questionnaire Clinical Risk Indicators for Children Development (URDI) and the Modified Checklist for Autism in Toddlers (M-CHAT) to detect psychic risks and investigate the possible relationship between psychic risk and alterations in cognitive development and language acquisition. Twenty children, between one and 24 months old, were evaluated in a cohort research by IRDI (from incomplete 4 months till 18 months), M-CHAT and Bayley III (18 and 24 months). There was a significant statistical correlation $(p=0.004)$ between language alteration in Bayley III and M-CHAT at 18 months. Children with psychic risk (IRDI-18 months) and low performance in Bayley III were reported. Although lack of concordance between IRDI and M-CHAT was detected, analysis between risk in M-CHAT and language alterations by Bayley III scale evidenced deep linguistic limitations in autism risk cases.
\end{abstract}

KEY WORDS: Child Development; Cognition; Language; Risk; Autistic disorder. 


\section{INTRODUÇÃO}

Estudos do campo psicanalítico e das neurociências evidenciaram a importância do acompanhamento do psiquismo na puericultura ${ }^{1-9}$ de modo a permitir que uma intervenção a tempo seja oportunizada ao bebê e sua família antes da instalação de uma psicopatologia, sobretudo em casos de risco de evolução para o autismo ${ }^{1-4}$. Esses estudos consideraram simultaneamente a possibilidade de predisposição biológica do bebê e de limitações na constituição do laço familiar.

Outros estudos da psicologia do desenvolvimento e das neurociências também abordam o tema, destacando a importância da intervenção no primeiro ano de vida para que as condições psíquicas e do desenvolvimento infantil possam ser modificadas positivamente impedindo o déficit cognitivo em bebês com risco de autismo5 Em ambos os campos, ressalta-se a importância de que profissionais da puericultura se apropriem de conhecimentos acerca da detecção precoce de sinais de risco psíquico ${ }^{2,6,7,8}$ sobretudo para autismo como sugere o estudo de Flores e Smeha ${ }^{9}$ na realidade brasileira.

Sabe-se que as dificuldades prolongadas na estruturação psíquica podem ter como consequências limitações cognitivas e linguísticas, como já relatado em trabalhos sobre autismo ${ }^{1,10,11,12}$, além do que vários estudos apontam sobre as limitações sociais e no desenvolvimento humano que podem advir na psicose.

Esta pesquisa pretende abordar este tema ao analisar casos de risco psíquico que incluem o risco na direção das duas psicopatologias: autismo e psicose, por meio do uso dos Indicadores Clínicos de Referência (Risco) ao Desenvolvimento Infantil (IRDI) ${ }^{2,6}$, elaborados a partir da teoria psicanalítica por um grupo de pesquisadores universitários brasileiros, em cujo conteúdo encontra-se um conjunto de 31 indicadores clínicos que revelam a probabilidade de aparecimento de distúrbios psíquicos posteriores $^{2,6}$.

Em referência aos indicadores clínicos abordados pela psicanálise, é especialmente no âmbito da saúde mental infantil que a sua utilização pode alcançar um emprego significativo. A justificativa da produção deste tipo de instrumento clínico sustentado nessa prática e teoria se dá porque a psicanálise tem colaborado para a compreensão das estruturas e dos padrões de funcionamento dos transtornos emocionais, comportamentais e dos atrasos no desenvolvimento, proporcionando dispositivos técnicos e maneiras de intervir que têm otimizado consideravelmente os atendimentos e as condições da vida dos sujeitos ${ }^{1-4}$.

Especificamente em relação ao risco para autismo, vários estudos têm abordado roteiros e sinais utilizáveis no primeiro ano de vida ${ }^{7,8,12}$. De modo especial os Sinais PREAUT $^{7,8}$, idealizados para detecção precoce de risco de autismo apresentaram-se em razoável concordância com o Modified Checklist for Autism in Toddlers (M-CHAT) ${ }^{12}$.

Considerando o fato de os sinais PREAUT possuírem a mesma base epistemológica do IRDI, a teoria freudo-lacaniana, neste estudo tem-se como objetivos analisar a concordância entre IRDI e M-CHAT na detecção de risco psíquico, bem como investigar a possível relação entre risco psíquico e alterações no desenvolvimento cognitivo e na aquisição da linguagem em um grupo de crianças acompanhadas em um estudo de coorte desde um mês de idade e que apresentaram risco psíquico em seu histórico por meio da análise do IRDI.

\section{METODOLOGIA}

Trata-se de um estudo de coorte retrospectivo, com acompanhamento longitudinal de bebês e análise quantitativa do desfecho do desenvolvimento.

A amostra deste estudo constou de 20 bebês nas faixas etárias de 18 e 24 meses que faziam parte de uma pesquisa maior denominada "Análise Comparativa do Desenvolvimento de Bebês Prematuros e a Termo, com e sem risco psíquico: da detecção à intervenção", coordenada pela orientadora deste trabalho, aprovada no Comitê de Ética em Pesquisa, sob número 652.722. Os responsáveis, contatados no setor de puericultura de um hospital universitário e no setor de Teste do Pezinho de uma unidade básica de saúde, quando seu bebê tinha até 30 dias de vida, foram esclarecidos sobre os objetivos e procedimentos da pesquisa e assinaram o termo de consentimento livre e esclarecido. Responderam a uma entrevista, que ofereceu dados psicossociais, obstétricos 
e sociodemográficos, e se comprometeram a trazer seus bebês para avaliações até os dois anos de vida.

As avaliações realizadas utilizadas nesta pesquisa por faixa etária foram:

i. Faixa etária 1- 3 meses e 1 dia a 4 meses incompletos - IRDI fase I;

ii. Faixa etária 2 - 6 meses e 1 dia a 7 meses e 29 dias - IRDI fase II;

iii. Faixa etária 3 - 8 meses e 1 dia a 9 meses e 29 dias - IRDI fase III;

iv. Faixa etária 4 - 17 meses e 1 dia a 18 meses e 29 dias - IRDI fase IV, MCHAT, Bayley III;

v. Faixa etária 5 - 23 meses e 1 dia a 24 meses e 29 dias - M-CHAT e Bayley III.

O IRDI foi observado durante as interações mãebebê e também na entrevista com as mães; 0 instrumento M-CHAT foi aplicado nas mães e conferido na observação das crianças; e o teste Bayley III foi aplicado por fisioterapeuta com formação para a aplicação do mesmo quando os bebês tinham 24 meses. Para análise dos resultados foram utilizados os critérios que demonstram risco em cada um dos instrumentos, nas faixas etárias elegidas para o estudo, identificando, assim, os bebês com risco. No caso do roteiro IRDI, tem-se risco quando dois ou mais indicadores estiverem ausentes na análise das quatro fases, ou seja, de um a 18 meses de vida.

O M-CHAT é capaz de detectar risco para autismo aos 18 e 24 meses. Ele é uma alteração do Checklist for Autism in Toddlers (CHAT), elaborado nos Estados Unidos e tem tradução para o português ${ }^{12}$. A aplicabilidade do M-CHAT é bastante acessível, sendo que as respostas a cada item consideram a opinião dos pais em correspondência ao comportamento da criança. Sua versão traduzida e adaptada foi publicada para o português após cauteloso estudo e aplicação a fim de testar sua eficácia. Apesar de a escala não apresentar validade de diagnóstico, pode indicar casos suspeitos que necessitam de uma avaliação mais minuciosa, pois apresenta alta sensibilidade e especificidade. De um total de 23 questões, 19 itens são relativos à presença/ausência de competências e quatro itens estão associados a comportamentos atípicos. Quando a criança avaliada apresenta um desenvolvimen- to típico, a devolutiva dada pelos pais às questões que indicam a presença de competências sociais ou do desenvolvimento normalmente é sim, já para os itens que avaliam a presença de comportamentos atípicos, tem-se habitualmente como resposta o não ${ }^{12}$.

O Bayley III (BayleyScalesofinfantandToddlerDevelopment, ThirdEdition), que foi publicado em 2006, busca avaliar o desenvolvimento infantil. Em 1993, Nancy Bayley e colaboradores realizaram os primeiros estudos sobre a escala Bayley, que é classificada como detentora de propriedades psicométricas para a avaliação do desenvolvimento infantil. Essa versão mais atual apresenta melhora do conteúdo dos testes, maior qualidade psicométrica e, em decorrência, maior adequação clínica. A aplicação do teste Bayley III é indicada para crianças de 1 a 42 meses de idade ${ }^{13}$.

A escala proporciona avaliação de múltiplos setores do desenvolvimento infantil, podendo ser utilizada para o propósito de identificar atrasos e fornecer informações para o planejamento terapêutico. A criança é observada quanto à execução de determinadas atividades esperadas nas faixas etárias. São avaliados cinco domínios de desenvolvimento: cognição, linguagem (comunicação receptiva e expressiva), motor (fino e grosso), socioemocional e comportamento adaptativo. Os três primeiros são analisados por meio da observação direta da criança durante o teste, e os dois últimos são analisados por intermédio de um questionário a ser respondido pelo principal cuidador da criança. Essas duas escalas são menos utilizadas em circunstâncias clínicas e de pesquisa na realidade brasileira porque ainda não foram traduzidas para o português e adaptadas à realidade nacional ${ }^{13}$.

O subteste cognitivo e o subteste de linguagem da Bayley III foram utilizados nesta pesquisa. Eles examinam o progresso da criança na cognição em relação as áreas como visualização, memória e atenção; e, na linguagem, avaliam as habilidades de comunicação receptiva e expressiva, incluindo a capacidade de reconhecer sons e vocabulário receptivo. O Bayley III não fornece uma pontuação global total, mas separa as pontuações brutas e escalares para cada domínio, bem como as pontuações compostas e as classificações percentuais para cada escala. Ao fim do teste, o desenvolvimento da criança pode 
receber uma das seguintes classificações: extremamente baixo, limítrofe, média baixa, média alta, superior e muito superior ${ }^{13}$.

Os dados normativos de Bayley III foram coletados nos Estados Unidos, em 2004, com 1.700 crianças com idades entre 16 dias e 43 meses e 15 dias. Os coeficientes de confiabilidade para os subtestes Bayley III são 0,86 , para motor fino; 0,87 , para comunicação receptiva; e 0,91 , para comunicação expressiva, cognição e motor grosso. Cada item do teste pode receber a pontuação 0 ou 1: 0 , caso a criança não realize $o$ item ou falhe em cumprir os parâmetros estabelecidos pelo teste; e 1 sendo a resposta alcançada quando a criança realiza os parâmetros estabelecidos, apresentando desempenho apropriado no item.

As regras de aplicabilidade do teste se constituem em encontrar a base do teste quando a criança for capaz de acertar em sequência três questões; se a criança responde erroneamente uma dessas três primeiras, retorna a letra anterior da testagem; continuando até que a criança erre cinco questões consecutivamente ${ }^{13}$. Para que a criança apresente alguma alteração cognitiva ou de linguagem, deve obter, como resultado, escore composto menor que 90 nesses subtestes.
Os resultados obtidos foram tabulados em planilha Excel e as análises estatísticas foram realizadas no software STATA 9.0, no qual se buscou a análise de concordância entre os protocolos IRDI e M-CHAT na verificação de risco psíquico, e a correlação e concordância entre IRDI, M-CHAT e Bayley III para verificar a relação entre risco psíquico e alteração cognitiva e/ou de linguagem.

\section{RESULTADOS}

Neste estudo foram analisados os resultados obtidos nas avaliações de um grupo de 20 bebês, avaliados pelo IRDI nos 18 meses iniciais de vida, buscando verificar os níveis de concordância e correlação entre a variável risco psíquico e o desfecho cognitivo, obtido pelo protocolo Bayley III e risco para autismo, avaliado pelo M-CHAT aos 18 e 24 meses.

Quando realizada essa relação por meio do teste de KAPPA, observou-se, conforme descrito na Tabela 1, que não houve concordância significativa entre IRDI e M-CHAT.

Tabela 1. Concordância IRDI e M-CHAT aos 18 e 24 meses

\begin{tabular}{ccccccccc}
\hline \multirow{2}{*}{ IRDI } & \multicolumn{4}{c}{ M-CHAT 18 meses } & \multicolumn{4}{c}{ M-CHAT 24 meses } \\
\cline { 2 - 9 } & Sem risco & Com risco & Total & Kappa & Sem risco & Com risco & Total & Kappa \\
\hline Sem risco & 8 & 1 & 9 & & 9 & 0 & 9 & $\mathrm{~K}=0,083$ \\
Com risco & 7 & 4 & 11 & $\mathrm{~K}=0,238$ & 10 & 1 & 11 & $\mathrm{p}=0,353$ \\
Total & 15 & 5 & 20 & & 19 & 1 & 20 & \\
\hline
\end{tabular}

Legenda: IRDI-Indicadores de Risco para Desenvolvimento Infantil; M-CHAT-Modified Checklist for Autism in Toddlers; K-coeficiente de correlação Kappa; p- valor de p.

É interessante observar que o IRDI identifica 11 crianças com risco aos 18 meses e o M-CHAT apenas cinco, demonstrando a sensibilidade de detecção maior ao risco psíquico por parte do IRDI, tendo em vista que ele não objetiva identificar apenas as crianças com risco para autismo, e considera a possibilidade de risco de constituição para psicose, ou mesmo risco ao desenvolvimento. Entre as alterações ao desenvolvimento, pode ser citado o atraso na aquisição da linguagem como uma das alterações mais frequentes na amostra estudada, como se verá na Tabela 4 em que dez crianças apresentam atraso de linguagem já aos 18 meses na avaliação do Bayley III.

$\mathrm{Na}$ faixa etária de 24 meses, apenas uma criança foi identificada com risco para autismo por meio do protocolo M-CHAT, ou seja, somente uma dentre o total de 11 crianças identificadas pelo IRDI como possuindo risco psíquico, o que explica a falta de concordância estatística entre ambos pelo fato de o IRDI pegar uma gama maior de fatores de risco para a constituição psíquica do que o M-CHAT. 
Nas Tabelas 2 e 3 são apresentadas as correlações entre IRDI e Bayley III. Na Tabela 2, encontram-se as análises de cognição e linguagem por meio da comparação de Spearman e na Tabela 3 apenas a correlação com a linguagem por meio do Teste U de Mann-Whitney.

Tabela 2. Correlação IRDI e Bayley III cognição e linguagem 18 e 24 meses

\begin{tabular}{lccc}
\hline & Valid & Spearman & p-level \\
\hline $\begin{array}{l}\text { IRDI x Bayley III } \\
\operatorname{cog} 24 \text { meses }\end{array}$ & 20 & 0,227 & 0,334 \\
$\begin{array}{l}\text { IRDI x Bayley III } \\
\text { cog18 meses }\end{array}$ & 20 & 0,267 & 0,254 \\
$\begin{array}{l}\text { IRDI x Bayley III } \\
\text { lgg 18 meses }\end{array}$ & 20 & 0,175 & 0,461 \\
$\begin{array}{l}\text { IRDI x Bayley III } \\
\text { lgg 24meses }\end{array}$ & 20 & 0,045 & 0,851 \\
\hline
\end{tabular}

Legenda: IRDI - Indicadores Clínicos de Referência (Risco) ao Desenvolvimento Infantil. BAYLEY III (Bayley Scales of infant and Toddler Development, Third Edition). COG: cognitivo. LGG: linguagem. Teste correlação de Spearman.

Tabela 3. Análise de comparação entre Bayley III Linguagem aos 18 e 24 meses e IRDI

\begin{tabular}{|c|c|c|c|}
\hline \multirow[t]{2}{*}{ BAYLEY III } & $\begin{array}{c}\text { IRDI } \\
\text { SEM RISCO }\end{array}$ & $\begin{array}{c}\text { IRDI } \\
\text { COM RISCO }\end{array}$ & \multirow{2}{*}{ P VALOR } \\
\hline & Média \pm DP & Média \pm DP & \\
\hline \multicolumn{4}{|l|}{18 MESES } \\
\hline Linguagem & $\begin{array}{c}94,667 \pm \\
23,388\end{array}$ & $\begin{array}{c}79,818 \pm \\
21,104\end{array}$ & 0,175 \\
\hline $\begin{array}{l}\text { Linguagem } \\
\text { receptiva }\end{array}$ & $\begin{array}{c}21,556 \pm \\
7,230\end{array}$ & $\begin{array}{c}17,091 \pm \\
8,264\end{array}$ & 0,370 \\
\hline $\begin{array}{l}\text { Linguagem } \\
\text { expressiva }\end{array}$ & $\underset{7,433}{22,333 \pm}$ & $\begin{array}{c}17,909 \pm \\
5,718\end{array}$ & 0,260 \\
\hline \multicolumn{4}{|l|}{24 MESES } \\
\hline Linguagem & $\begin{array}{c}93,556 \pm \\
15,322\end{array}$ & $\begin{array}{c}89,000 \pm \\
14,920\end{array}$ & 0,602 \\
\hline $\begin{array}{l}\text { Linguagem } \\
\text { receptiva }\end{array}$ & $\begin{array}{c}24,333 \pm \\
4,359\end{array}$ & $\underset{4,262}{23,182 \pm}$ & 0,551 \\
\hline $\begin{array}{l}\text { Linguagem } \\
\text { expressiva }\end{array}$ & $\begin{array}{c}27,000 \pm \\
6,982\end{array}$ & $\begin{array}{c}23,091 \pm \\
8,154\end{array}$ & 0,175 \\
\hline
\end{tabular}

Legenda: IRDI - Indicadores Clínicos de Referência (Risco) ao Desenvolvimento Infantil; BAYLEY III - Bayley Scales of infant and Toddler Development, Third Edition; Teste U de Mann-Whitney.

Em termos descritivos, é interessante observar que, apesar da ausência de significância estatística na associação IRDI e Bayley III para cognição e linguagem, que há uma média maior de pontuação em linguagem para crianças sem risco psíquico que seja ser de 14 pontos aos 18 meses e diminui aos 24 meses para 4 pontos.

Na Tabela 4, há uma descrição dos resultados, utilizando o Teste U de Mann-Whitney para comparação entre M-CHAT e Bayley III Linguagem aos 18 meses e 24 meses.

Tabela 4. Análise comparativa entre Bayley III Linguagem e M-CHAT aos 18 e 24 meses $(\mathrm{N}=20)$

\begin{tabular}{|c|c|c|c|}
\hline VARIÁVEIS & $\begin{array}{c}\text { M-CHAT } \\
\text { SEM RISCO }\end{array}$ & $\begin{array}{c}\text { M-CHAT } \\
\text { COM RISCO }\end{array}$ & $\begin{array}{c}\mathbf{p} \\
\text { valor }\end{array}$ \\
\hline $\begin{array}{l}\text { BAYLEY III } \\
18 \text { meses }\end{array}$ & Média \pm DP & Média \pm DP & \\
\hline Linguagem & $\begin{array}{c}94,467 \pm \\
19,744\end{array}$ & $62,600 \pm 12,798$ & $0,004^{*}$ \\
\hline $\begin{array}{l}\text { Linguagem } \\
\text { receptiva }\end{array}$ & $\begin{array}{c}21,600 \pm \\
6,610\end{array}$ & $11,600 \pm 7,301$ & $0,012^{*}$ \\
\hline $\begin{array}{l}\text { Linguagem } \\
\text { expressiva }\end{array}$ & $\underset{5,858}{22,200 \pm}$ & $13,000 \pm 4,123$ & $0,011^{*}$ \\
\hline \multicolumn{4}{|l|}{$\begin{array}{l}\text { BAYLEY III } \\
24 \text { meses }\end{array}$} \\
\hline Linguagem & $\begin{array}{c}91.666 \pm \\
15,327\end{array}$ & $85,500 \pm 12,020$ & 0,448 \\
\hline $\begin{array}{l}\text { Linguagem } \\
\text { receptiva }\end{array}$ & $\begin{array}{c}24,000 \pm \\
4,144\end{array}$ & $21,000 \pm 5,656$ & 0,311 \\
\hline $\begin{array}{l}\text { Linguagem } \\
\text { expressiva }\end{array}$ & $\begin{array}{c}24,833 \pm \\
8.125\end{array}$ & $25,000 \pm 2,828$ & 0,704 \\
\hline
\end{tabular}

Legenda: BAYLEY III - Bayley Scales of infant and Toddler Development, Third Edition;. M-CHAT - Modified Checklist for Autism in Toddlers; Teste U de Mann-Whitney; *p valor $\leq 0,05$.

Observa-se que houve significância estatística aos 18 meses na relação entre ter risco no M-CHAT e atraso na aquisição da linguagem no Bayley III, tanto na modalidade receptiva, quanto expressiva da linguagem, o que evidencia o observado na Tabela 3 , com um pouco mais de peso estatístico.

Embora a diferença na média e no desvio-padrão entre as crianças sem e com risco psíquico não tenha levado a um valor significativo, observa-se que a média no desempenho geral de linguagem das crianças sem risco é sempre superior, tanto aos 18, quanto aos 24 meses. Também se destaca a ocorrência de um desvio-padrão maior do grupo com risco em termos de linguagem expressiva aos 24 meses. Esse dado se deve ao fato de que esse sintoma de linguagem é considerado como o mais evidente nas alterações do desenvolvimento, em termos de desfecho dos bebês com risco psíquico. 


\section{DISCUSSÃO}

Considerando a proposta inicial de analisar a relação entre desenvolvimento cognitivo e de linguagem em sua possível associação com risco psíquico, observouse que o risco psíquico nem sempre foi acompanhado de risco ao desenvolvimento cognitivo e linguagem, o que explica a falta de correlação estatística entre os resultados obtidos no Bayley III para os itens de linguagem e cognição aos 18 e 24 meses, e os resultados obtidos no roteiro IRDI nos 18 primeiros meses de vida. Uma hipótese que se pode sugerir é que há, no segundo ano de vida, uma diferenciação maior entre os aspectos estruturais, ou seja, entre cognição e psiquismo.

A relação encontrada entre o M-CHAT e o Bayley III, em seu aspecto linguagem, demonstra o que diversos trabalhos apontaram sobre o evidente comprometimento da linguagem em casos de autismo ${ }^{4,5,10,11,12}$. Assim, pode-se concluir que a alteração de linguagem é mais evidente do que a cognitiva no segundo ano de vida, já que as crianças com risco psíquico apresentaram desenvolvimento cognitivo típico, em sua maioria. Essa relação entre risco psíquico e alteração de linguagem foi encontrada em amostra maior e não restrita apenas ao autismo, no trabalho de Crestaniet al. ${ }^{14}$, que evidenciou relação entre menor produção de fala inicial e risco avaliado pelo IRDI.

No entanto, cabe ressaltar que a continuidade do déficit linguístico coloca, como possibilidade futura, a presença de dificuldades no âmbito cognitivo, já que as tarefas cognitivas ficam mais complexas com o avançar da idade e demandam um funcionamento de linguagem ampliado para sua compreensão. Esse aspecto ganha maior relevância quando se observa a tendência de melhor desempenho linguístico nas crianças sem risco psíquico, quando comparadas com as com risco, embora não tenha havido uma significância estatística. Esse aspecto é ressaltado por Polyaket al. ${ }^{11}$ ao abordarem a necessidade de se investigar mais o status da cognição em casos de autismo. Os autores afirmam que o déficit cognitivo é uma comorbidade comum no autismo. Esse fato põe em maior evidência a necessidade de detecção precoce do autismo para que a intervenção precoce possa sustentar melhores condições de evolução linguística e cognitiva para a criança com esta psicopatologia.
Outro aspecto a ser considerado, a partir dos resultados desta pesquisa, é a necessidade nosográfica da psicose enquanto quadro estrutural do psiquismo, visto que o IRDI evidenciou a possibilidade de detectar a presença de risco psíquico em vários casos em que não foi possível pelo M-CHAT. Esse fato foi observado pela falta de concordância entre IRDI e M-CHAT, porque este teste possui maior especificidade para autismo ${ }^{12}$, enquanto que o IRDI ${ }^{2,6}$ caracteriza-se por ser um roteiro que capta outros tipos de risco psíquico, como, por exemplo, a estruturação psicótica ${ }^{1}$. Pode-se observar, ainda, que o sintoma de linguagem foi o mais comum na relação com o risco psíquico detectado pelo IRDI, embora não de modo estatístico. Esses resultados evidenciam que as alterações de linguagem devem ser investigadas em conjunto com o psiquismo.

Um aspecto importante a se ressaltar sobre o roteiro IRDI ${ }^{2,6}$ é o fato de ele analisar a dinâmica da relação parental com o bebê e não apenas os sinais patológicos que o bebê apresenta. Isso o diferencia muito do M-CHAT porque amplia o olhar em termos nosográficos, mas principalmente na direção da abordagem de intervenção em tempo que será feita. Ao observar os eixos teóricos de estabelecimento da demanda, suposição do sujeito, alternância entre presença e ausência e função paterna, consegue-se perceber as dificuldades no laço entre bebê e pais e não apenas as fragilidades biológicas que o bebê apresenta para engajar-se na relação com aqueles que exercem as funções parentais. Essas observações podem, portanto, criar um contexto clínico da direção da intervenção e não apenas dizer da presença ou ausência de risco.

\section{CONCLUSÃO}

Considerando o objetivo inicial de avaliar a concordância entre IRDI e M-CHAT, observou-se a ausência de concordância entre ambos os instrumentos.

Por outro lado, observou-se que a análise entre risco no M-CHAT e alterações de linguagem na escala Bayley III evidenciaram as graves limitações linguísticas em casos de risco para autismo. 


\section{REFERÊNCIAS}

1. Laznik MC, Touati B. Distinção clínica e teórica entre autismo e psicose na infância. São Paulo: Instituto Langage; 2016.

2. Jerusalinsky A. Indicadores de risco: como a psicanálise pode proteger os bebês. In: Jerusalinsky A. (Org.) Dossiê autismo. São Paulo: Instituto Langage; 2015. p. 418-33.

3. Laznik MC, Pelabon C. Caso clínico de um bebê a risco de autismo: Leo e "la vraie vie". In: Parlato-Oliveira E, Cohen D. (Org.). O bebê e o outro: seu entorno e suas interações. São Paulo: Instituto Langage; 2017. p. 159-70.

4. Ambrós TMB, Correa AN, Oliveira LD, Souza APR. A musicalização como intervenção precoce junto a bebê com risco psíquico e seus familiares. Rev. latinoam. psicopatol. fundam, 2017;20(3):560-78.

5. Trevarthen C, Delafield-Butt JT. Autism as a developmental disorder in intentional movement and affective engagment. Front Integr Neurosci, 2013;7(49):1-16.

6. Kupfer MCM, Jurusalinsky AN, Bernardino LMF, Wanderley D, Rocha PSB, Molina SE, et al. Valor preditivo de indicadores clínicos de risco para o desenvolvimento infantile: um estudo a partir da teoria psicanalítica. Lat .Am. Journal of Fund. Psychopath, 2009;6(1):48-68.

7. OUSS L, Saint-Georges C, Robel L, Bordeau N, Laznik MC, Crespin GC, et al. Infants engagment and emotion predictors of autism or intelectual disability in West Syndrome. Eur. Child Adolsc. Psychiatry, 2014; 23(3):143-9.

8. OLLIAC B, Crespin G, Laznik MC, El Ganouni OCI, Sarradet JL, Bauby C, et al. Infant and dyadic assessment in early community-based screening for autism spectrum disorder with PREAUT grid. PLOS ONE, 2017, 0188831:1-22.

9. Flores MR, Smeha LN. Bebês com risco de autismo: o
nãoolhardomédico.Ágora,2013;16(especial):141-57.

10. Tamanaha AC, Mateleto MRF, Perissionoto J. The interference of expressive language status on autism behaviour check list scores in verbal and non-verbal autistic children. Audiol Commun Res., 2014; 19(2):167-70.

11. Polyak A, Kubina RM, Girirajan S. Comorbity of intelectual disability confounds ascertainment of autism: implications for genetic diagnosis. American J Med Genet, Neuropsychiat Genet, 2015;part B( 9999):1-9.

12. MURATORI, F. O diagnóstico precoce no autismo: guia prático para pediatras. Salvador: Núcleo Interdisciplinar de Intervenção Precoce da Bahia; 2014. 89p.

13. Bayley Scales of Infant and Toddler Development. Third edition. Administration Manual. San Antonio, TX: The Psychological Corporation; 2006.

14. Crestani AH, Moraes AB, Souza APR. Análise da associação entre índices de risco ao desenvolvimento infantil e produção inicial de fala entre 13 e 16 meses. Rev. CEFAC, 2015;17(1):169-76.

Recebido em: 11/03/2018 Aceito em: 06/06/2018 